\title{
Design and Optimisation of a Three Layers Thermal Neutron, Fast Neutron and Gamma- Ray Imaging System
}

\author{
H. Al Hamrashdi, S. D. Monk, and D. Cheneler \\ (Lancaster University, Engineering Department)
}

\begin{abstract}
The design and configuration of a multilayered imaging system with the ability to detect thermal neutrons, fast neutrons and gamma rays has been developed and its efficacy demonstrated. The work presented here numerically determines the systems efficiency and spatial resolution, using ${ }^{252} \mathrm{Cf}$ and ${ }^{137} \mathrm{Cs}$ as a case study. The novelty of this detection system lies in the use of small form factor detectors in a three-layer design, which utilises neutron elastic scattering and Compton scattering simultaneously. The current configuration consists of $10 \mathrm{~mm}$ thick natural lithium glass (GS10) scintillator integrated with a $20 \mathrm{~mm}$ thick plastic scintillator (EJ-204) in the first layer, a $15 \mathrm{~mm}$ thick lithium glass (GS10) scintillator in the second and a $30 \mathrm{~mm}$ thick CsI(TI) scintillator forming the final layer. Each of these layers is backed with an $8 \times 8$ silicon photomultiplier diode (SiPM) array. The overall size of the imaging system is 27 mm x $27 \mathrm{~mm}$ x $135 \mathrm{~mm}$. MCNPv6.1 and Geant4-10.04 were alternatively used to optimise the overall configuration and to investigate detection modalities. Results show promising performance with high precision source localisation and characterization abilities. Measurements were virtually obtained of two gamma-ray sources within steel enclosures at angles of $15^{\circ}, 30^{\circ}$ and $50^{\circ}$ separation in order to test spatial resolution ability of the system. With the current active size of the system and the 8x8 SiPM configuration, the results estimate the spatial resolution to be close to $30^{\circ}$. The ability of the system to characterise and identify sources based on the type and energy of the radiation emitted, has been investigated and results show that for all radiation types the system can identify the source energy within the energy range of typical reported sources in literature.
\end{abstract}

Keywords - Neutrons, Gamma-rays, Lithium glass, EJ-204, CsI(TI), Nuclear Materials Security, MCNPv6.1, Geant4.

\section{INTRODUCTION}

The detection and localization of special nuclear materials (SNM) and radioactive materials in general, is a significant problem for national security agencies across the globe and international organisations such as the IAEA $[1,2]$. Over the last two decades, much research has involved the investigation of the design and the build of passive neutron/gamma mobile detection systems for safeguard and security applications [3]. The size of these systems range from vehicle scale down to wearable technology scale [4-10]. Many vehicle size systems, such as [4-6], are designed to detect strong-orphaned sources at long distances with a very wide field of view, although these systems have limited portability. Handheld devices offer an efficient solution for personal and luggage inspection at security checking points and within potentially crowded areas such as airports, but current systems have limited functionality. Commercially available handheld devices, such as Thermo Scientific ${ }^{\mathrm{TM}}$, PackEye Radiation Detection Backpack [11] and The Bruker Radiation Backpack Sentry [12] offer localisation abilities for gamma sources only, and have a relatively narrow field of view. To enhance systems abilities to identify and characterise radioactive materials and SNM, the focus on simultaneous detection of both neutrons and gamma-rays has grown noticeably in the last decade. An example of a handheld device that employs the characteristics of modern scintillation materials with dual particle detection abilities is a $\mathrm{Cs}_{2} \mathrm{LiYCl}_{6}$ : $\mathrm{Ce}$ (CLYC) based monitoring device [7] which claims radioisotope identification and localisation of thermal neutron and gamma-ray sources. Some coded aperture based imaging systems that are related to homeland security and safeguard applications with dual-mode capability offer enhanced sensitivity and source localization abilities. Examples include the three-dimensional localisation of radioactive sources using liquid scintillators [8] and the CLYC based RadCam imaging system which are based on the RadCam gamma imaging system [9]. Multi-layered dualparticles imaging systems use neutron scattering and Compton scattering techniques to offer a better field of view and higher detection efficiency $[10,13]$. For example, the dual-particle imager by Polack et al. [10] allows for accurate detection and source localisation even in the presence of shielding. This offers a larger range of detection materials, shorter acquisition time and a wider range of targets.

The aim of the work here is to design and optimise an innovative handheld, real-time dual-particle imaging system that is capable of simultaneously detecting thermal neutrons, fast neutrons and gamma rays using Monte Carlo simulations. The design utilises multi-layered system techniques to enhance the spectral and temporal abilities of the system, while keeping the weight and size flexible. The proposed design will allow the system to image, localise and 
characterise a wide range of radioactive sources and SNM in a real time frame. The system is capable of detecting three different types of radiation, allowing a wider range of radioactive sources and SNMs monitoring capabilities.

\section{IMAGING SYSTEM CONCEPT}

The multi-layered imaging system described here is based on the combination of three different modes of radiation detection in one handheld device: thermal neutron capture reactions in ${ }^{6} \mathrm{Li}$ nuclei, neutron scattering physics for fast neutrons detection and Compton scattering physics for gamma-ray detection. Scintillation crystal candidates along with their interaction probabilities and proposed thicknesses for a multi-particle detection system were extensively studied in previous work [14]. The work presented here goes beyond this earlier study in order to better understand the interaction of these detection subsystems and to verify the efficacy of their combination to form a more comprehensive radiation detection system.

The first layer consists of a $10 \mathrm{~mm}$ thick Lithium glass (GS10) scintillator (Scintacor Ltd) combined with a $20 \mathrm{~mm}$ thick EJ-204 plastic scintillator. The second layer features a $15 \mathrm{~mm}$ thick Lithium glass (GS10) scintillator (Scintacor $\mathrm{Ltd}$ ), with a $30 \mathrm{~mm}$ thick $\mathrm{CsI}(\mathrm{Tl})$ scintillator forming the final absorption layer. The rational behind the use of GS10 (natural Lithium content) rather than GS20 (95\% ${ }^{6} \mathrm{Li}$ content) in the first and second layers is mainly due to technical and financial reasons. GS10 will serve the purpose of proving the ability of the system to capture thermal neutrons with $10 \mathrm{~mm}$ thick crystal [15] and sufficiently scatter fast neutrons and gamma rays [14]. Secondly, GS10 was found to be more practical in the current prototype due to financial restrictions. It is assumed here that SensL's ArrayJ-30035-64P-PCB 8 x 8 $\mathrm{SiPM}$ is to be used as the photodetector throughout the design [16]. SiPM's are compact in size and require a relatively low operating voltage $(\sim 30 \mathrm{~V})$ offering a solution for small size imaging systems. This 64-pixel avalanche photodiode offers a segmented detection of pulses generated on the area covered by the detector. Hence, the geometry used to define the pixels in the simulation were based on these SiPMs. A schematic of the system arrangement is shown in figure 2.1 .

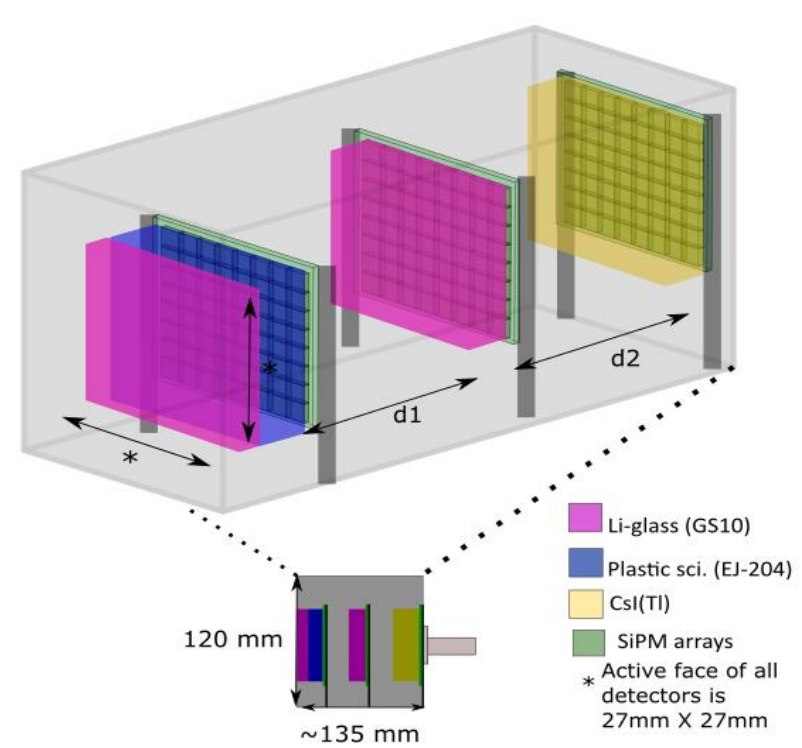

Fig. 2.1 Schematic of the multi-layered system configuration.

The thermal neutron detection mechanism in this imaging system is based on the ${ }^{6} \mathrm{Li}(\mathrm{n}, \alpha)^{3} \mathrm{H}$ capture reaction in the first layer lithium glass detector, releasing alpha and triton particles. Lithium glass will emit detectable light as a result of the energy deposited by the resultant ionising particles [17]. The thermal capture cross section for this particular reaction is 940 barns with a $\mathrm{Q}$ value of $+4.78 \mathrm{MeV}$. The aim of adding thermal neutron detection in the first layer is to enhance the energy range of detected neutron source. Although most SNM is characterised with neutrons in the fast energy range $(>1 \mathrm{keV})$, shielding around smuggled or hidden sources can moderate the energy of these fast neutrons to be in the thermal neutron energy range.

Fast neutron imaging in the system is based on the physics of neutron elastic scattering in hydrogen rich EJ-204, followed by a second elastic scattering event in the Li-glass detector. The principle of fast neutron imaging in scattering cameras is thoroughly discussed within the literature $[10,13$, 18-20]. The elastic scattering physics proportionally relates the neutron scattered angle in the first detector, $\theta$, with the energy of the recoil proton, $E p$ and the energy of the scattered neutron, $E n^{\prime}$ (equation 1)

$$
\tan ^{2} \theta=E p / E n^{\prime}
$$

The energy of the recoiled proton is measured in the first scattering plane. The energy of the scattered neutron is calculated using the time of flight $(T o F)$ between the two neutron scattering planes as illustrated in equation 2 .

$$
E n^{\prime}=\frac{1}{2} m\left[\frac{d_{1}}{T o F}\right]^{2}
$$

Where $m$ is the mass of the neutron and $d_{1}$ is the separation between the two scattering planes. The probability cone of the location of the source can be reconstructed using the scattering angle of each incident neutron. The intersection of the cones generated by all incident neutrons will form an 
image of the neutron source. Figure 2.2, shows a schematic of a neutron scattering event in the system.

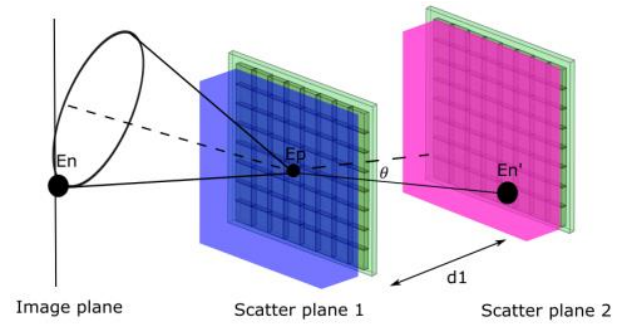

Fig. 2.2 Schematic of a neutron scattering system, with monolithic detectors and segmented photodetectors.

Gamma-ray detection in this system is based on Compton scattering kinematics; examples of the analysis of Compton camera in literature can be found abundantly [21, 22]. This method of gamma-ray detection has been studied extensively for the last four decades and has proved to offer a wider field of view and improved sensitivity over conventional mechanically collimated systems [23-25]. In principle, and as illustrated in figure 2.3, gamma rays scatter in the first plane depositing part of their energy before undergoing photoelectric absorption in a second high density scintillator. The energy and position of the two interactions are then utilised to find the angle between the incident and scattered gamma-rays, $\varphi$. Compton kinematics relates these parameters and allows the calculation of $\varphi$ as in equation 3 :

$$
\cos \varphi=1+0.511 \mathrm{MeV}\left[\frac{1}{E_{1}+E_{2}}-\frac{1}{E_{2}}\right]
$$

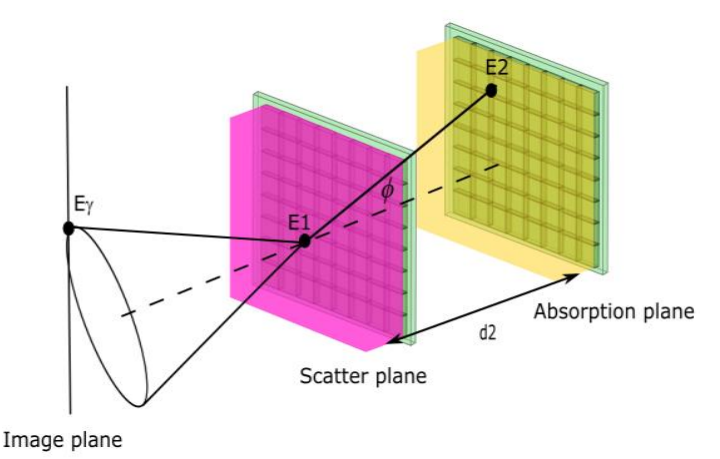

Fig. 2.3 Schematic of Compton scattering system with monolithic detectors and segmented photodetectors.

Where $\mathrm{d} 2$ is the separation between layers two and three. This will allow the generation of a probability cone of the incident gamma-ray location. The intersection of the cones created by all detected Compton scattering events is used to obtain the source location.

\section{PROTOTYPE SETUP}

MCNPv6.1 [26] and Geant4-10.04 [27] Monte Carlo codes have been used to investigate the optimum configuration of detectors and layers and then to test the feasibility and efficacy of this design. In the MCNP environment, neutron simulations utilised data from the ENDF70a file which is based on the ENDF/B-VII cross-section data [26]. The
Evaluated Photon Data Library (EPDL) and the Activation Library (ACTL) compilations from Livermore data libraries were mainly used in the simulation of gamma photon transport [26]. All default options in physics cards are applied except for the neutrons physics card where analog capture is invoked. In Geant4, additional gamma simulations used the Electromagnetic (EM) physics package. The model is based on the emstandard physics list with the Livermore evaluated library [27]. For all simulations a point source configuration was used in testing the response of the system. The exact position and energy of the point sources used in the simulations is investigation specific and details are discussed during each investigation in the following sections.

In the current design and for all simulations, all layers have the same active front face size of $27 \mathrm{~mm} \mathrm{x} 27 \mathrm{~mm}$. Simulations were first used to investigate the effect of increasing the separation distance between detection layers, $\mathrm{d} 1$ and $\mathrm{d} 2$, and detection efficiency of the system. For the neutron scattering layers, a bare ${ }^{252} \mathrm{Cf}$ source $1 \mathrm{~cm}$ away from the system was used in MCNP6.1.0 simulations with no intervening materials and no scattering objects. Although simulations started at separation distance $d 1=10 \mathrm{~mm}, 30 \mathrm{~mm}$ is the minimum $\mathrm{d} 1$ in the current porotype configuration because of the physical size of the SiPM array and the associated breakout board. The relationship between the numbers of successive scattering events relative to the total number of fast neutrons entering the system and the separation distance is plotted in figure 3.2. The efficiency decreases with increasing plane separation, with the maximum efficiency observed at a $30 \mathrm{~mm}$ plane separation.

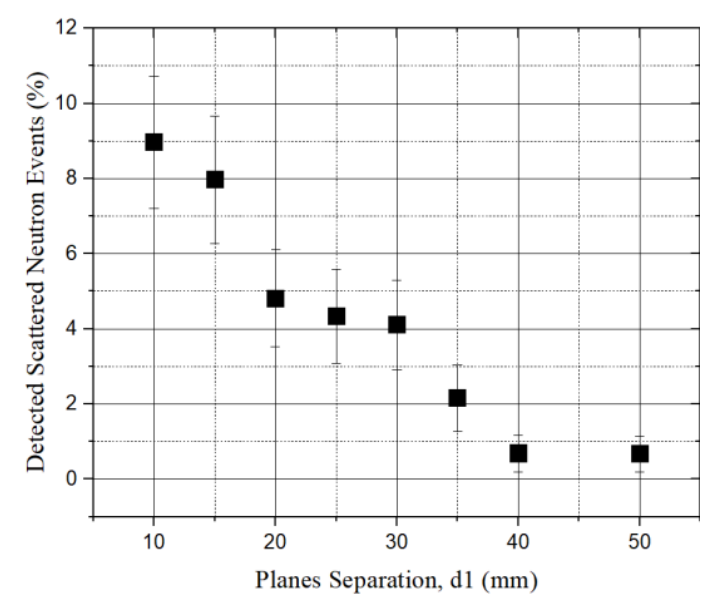

Fig. 3.2 Detected fast neutron scattering events as function of planes separation for ${ }^{252} \mathrm{Cf}$ source.

Optimising the value of $\mathrm{d} 2$ in the Compton camera subsystem was based on Geant4-10.04 simulations of four different gamma-ray energies in point source configuration with no intervening materials and no scattering objects. The relation between the fractions of Compton scattering events relevant to the total number of gamma rays entering the system was plotted in figure 3.3 as a function of the plane separation. As above, in reality the minimum plane separation starts at $30 \mathrm{~mm}$ because of the physical size of the SiPM and the associated breakout board in the current configuration. 
The efficiency decreases with increasing planes separation with maximum efficiency at $30 \mathrm{~mm}$.

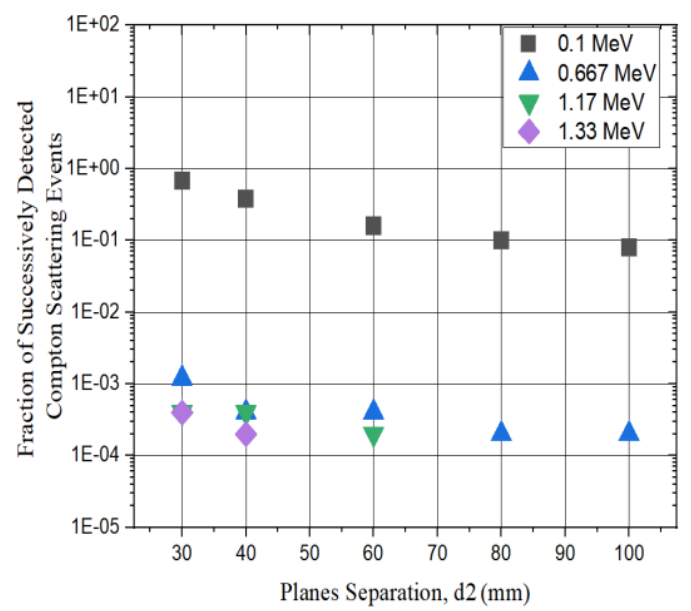

Fig. 3.3 Fraction of successively detected Compton events as function of planes separation.

The overall trend of the results shows that increasing the intra-plane distance between detectors for this multi-layered system decreases the probability of successively detected scattering events especially for fast neutrons. However, this flexible prototype configuration allows changing the size of the front face of layers which can consequently increases detection probabilities and improves geometric efficiency.

\section{SySTEMS RESPONSE AND DETECTION CHARACTERISTICS}

\subsection{Systems response to a thermal neutron source.}

Here, the ability of the system to locate and identify thermal neutron point sources in a $10 \mathrm{~cm} \mathrm{x} 10 \mathrm{~cm}$ carbon steel enclosure was investigated. The source was located $1 \mathrm{~cm}$ away from the system at the centre of the detector with no intervening materials and no scattering objects. An example of the simulation made in the MCNPv6.1 environment is shown in figure 4.1. The 2D figure shows a top down view of thermal neutrons tracks inside the box and their trajectory in the detector. The 3D right hand figure shows the distribution of particles inside the carbon steel box.
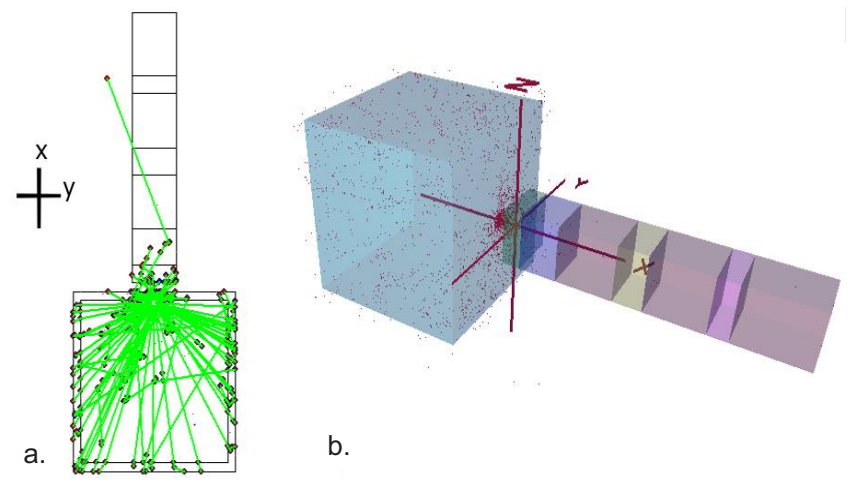

b.

Fig. 4.1 a. 2D and b. 3D representation of the MCNPv6.1 simulation of a bare thermal neutrons source.
The results in this section are mainly presented as reconstruction images of the system response in an $8 \times 8$ array format (the size of the SiPM photodetector utilised in the design), which epitomises the configure of light output of each layer.

Figure 4.2 show image reconstruction of the signal generated in the lithium glass detector in the first layer (MCNPv6.1.0). The calculated intrinsic efficiency was found to be $(79 \pm 3) \%$.

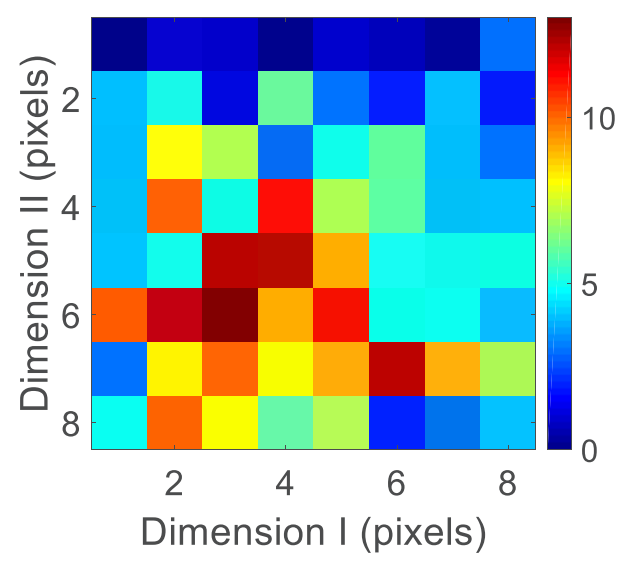

Fig. 4.2 System response to thermal neutron source located at pixels a. $(4,4) \mathrm{cm}$, colour bar shows pixel intensity.

\subsection{Systems response to fast neutron sources.}

The response of the system to fast neutrons is based on neutron scattering kinematics in EJ-204 within the first layer and lithium glass $(15 \mathrm{~mm})$ in the second layer. Using the PTRAC file generated in MCNPv6.1 simulations, energies of scattered fast neutrons and $T o F$ in these two layers was found. Based on equations (1) and (2), estimation of the source location was obtained. In the first testing scenario, the response of the system to a bare ${ }^{252} \mathrm{Cf}$ source placed at different positions was investigated in order to evaluate the detection ability of the system as well as the spatial resolution of the system. The source to detector distance was $1 \mathrm{~cm}$ with no intervening materials and no scattering objects. The results in figure 4.5 illustrate the ability of the system to identify the direction of incident radiation using neutron scattering kinematics. 

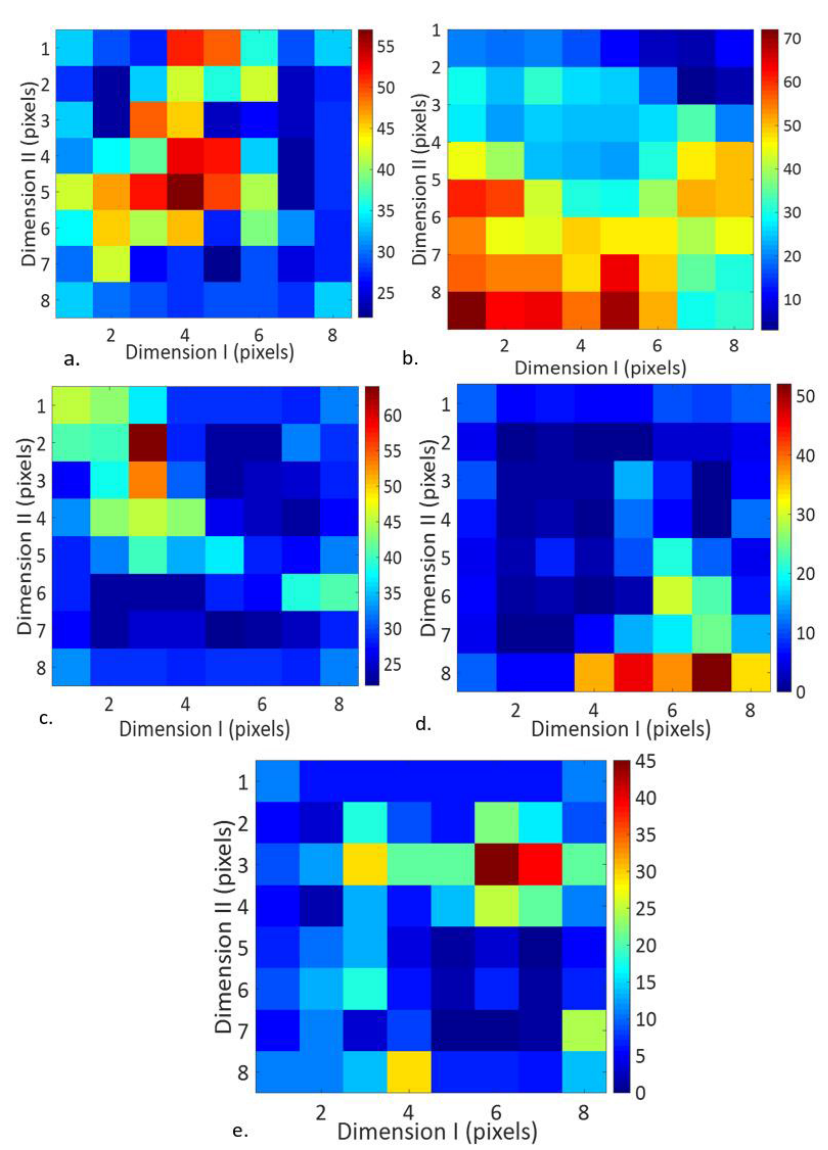

Fig. 4.5 The response of the system to a ${ }^{252} \mathrm{Cf}$ source located at five different positions at pixels a. $(4,4), b .(7,2)$ cm, c. $(2,2)$ d. $(7,7)$ e.(2,7) (Colour bars indicate image intensity).

The second test involved simulations utilising ${ }^{252} \mathrm{Cf}$ and ${ }^{241} \mathrm{AmBe}$ sources located $1 \mathrm{~cm}$ from the detection plane, in order to test the ability of the system to identify two fast neutron sources based on their energies. Table 4.1 list reported average energies and calculated average energies of these two sources. These results illustrate the system's ability to identify different neutron sources based on their energies.

Table 4.1 Systems average energy calculations for different fast neutrons sources $[17,28]$.

\begin{tabular}{|c|c|c|}
\hline Source & $\begin{array}{c}\text { Average Energy in } \\
\text { Literature (MeV) }\end{array}$ & $\begin{array}{c}\text { Average energy } \\
\text { calculated from } \\
\text { simulations (MeV) }\end{array}$ \\
\hline${ }^{241} \mathrm{AmBe}$ & 4.2 & $4.2 \pm 1.3$ \\
\hline${ }^{252} \mathrm{Cf}$ & 2.35 & $2.0 \pm 0.8$ \\
\hline
\end{tabular}

\subsection{Systems response to a gamma source.}

The ability of the system to identify the location of gamma sources was investigated using a bare ${ }^{137} \mathrm{Cs}$ point source. In the simulations (Geant4), the source was positioned $30 \mathrm{~cm}$ away from the system, with no intervening materials and no scattering objects. Using equation 3 where $\mathrm{E} 1$ and $\mathrm{E} 2$ are the energies deposited in Li-glass and $\mathrm{CsI}(\mathrm{Tl})$ respectively, probability cones were constructed and the image intensities map of SiPM was constructed as illustrated in figure 4.6. The results in this figure show a high ability to precisely locate the position of a gamma-ray source.
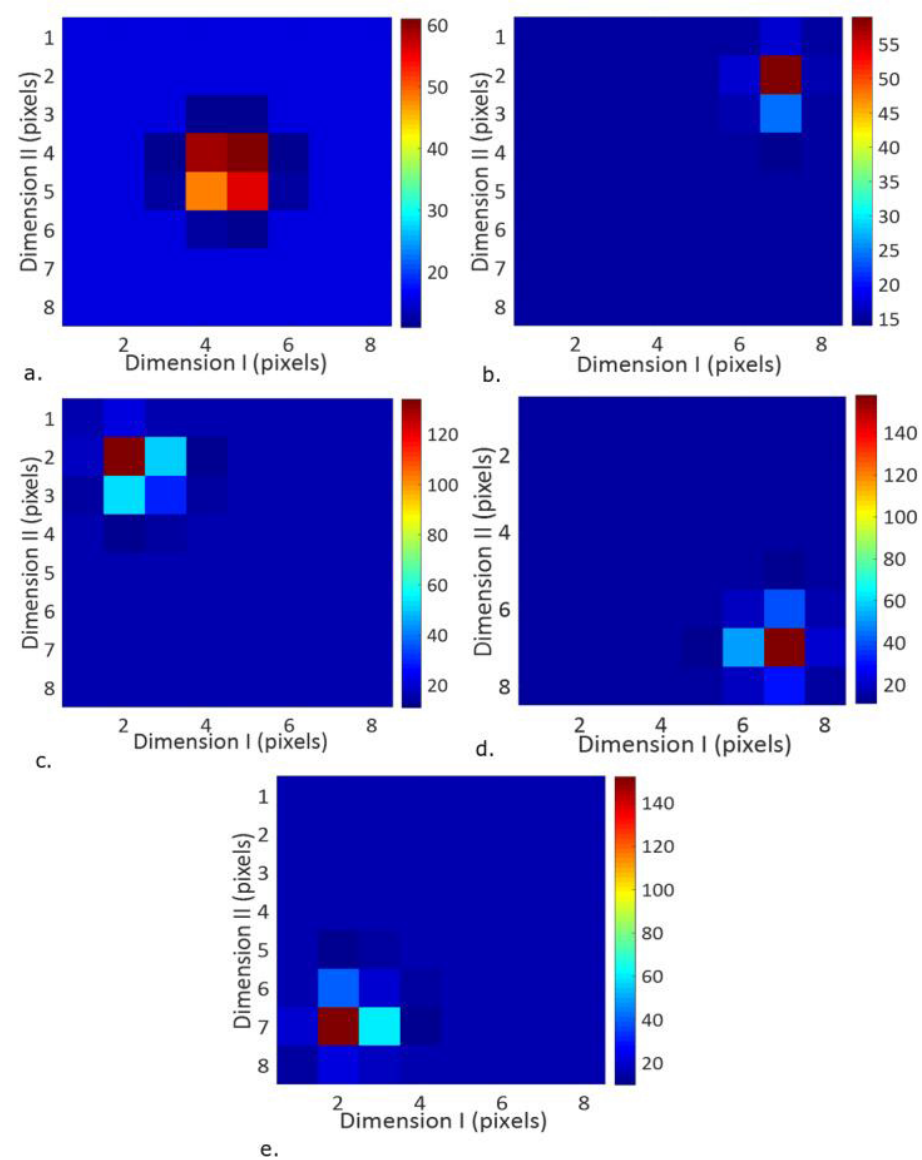

Fig. 4.6 The response of the system to a ${ }^{137} \mathrm{Cs}$ source located at five different pixel positions a. $(5,5), \mathrm{b} .(7,2)$, c. $(2,2)$ d. $(7,7)$ e. $(2,7)$ (Colour bars indicate image intensity).

In addition, the ability of the system to differentiate between closely spaced sources was investigated using two ${ }^{137} \mathrm{Cs}$ sources. The sources were placed $1 \mathrm{~mm}$ away from the imaging system. The results of this investigation are shown in figure 4.7 for $15^{\circ}, 30^{\circ}$ and $50^{\circ}$ sources separation, with spatial resolution close to $30^{\circ}$. 


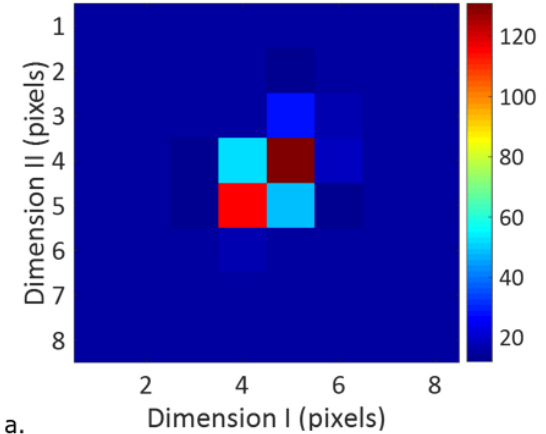

a.
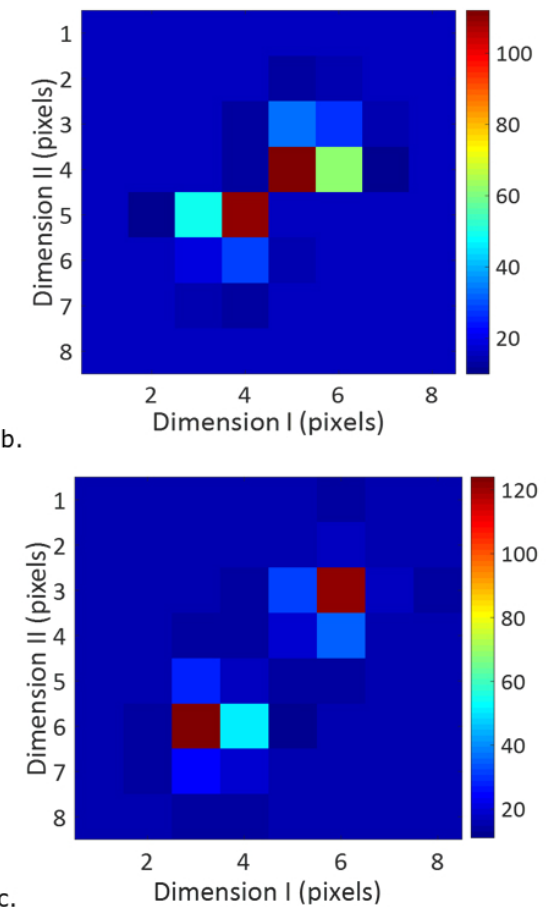

Fig. 4.7 System's response to two ${ }^{137} \mathrm{Cs}$ sources at a. $15^{\circ}$ and b. $30^{\circ}$ and c. $50^{\circ}$ separation distance (Colour bars indicate image intensity).

The system's capability to identify and characterise gamma-ray sources according to their energy was investigated using the simulations of ${ }^{137} \mathrm{Cs}$ and ${ }^{60} \mathrm{Co}$ point sources. The sources were placed $1 \mathrm{~mm}$ away from the system. Using equation 3 with E1 and E2 being the eneries deposited in Li-glass and $\mathrm{CsI}(\mathrm{Tl})$ respectively, the average gamma-ray energy peaks were calculated. Table 4.2 shows calculated average energies from simulations and reported average energies in literature.

\begin{tabular}{|c|c|c|}
\hline Source & $\begin{array}{c}\text { Average Energy in } \\
\text { Literature (MeV) }\end{array}$ & $\begin{array}{c}\text { Average energy } \\
\text { calculated from } \\
\text { simulations }(\mathrm{MeV})\end{array}$ \\
\hline${ }^{137} \mathrm{Cs}$ & 0.667 & $0.669 \pm 0.006$ \\
\hline${ }^{60} \mathrm{Co}$ & (1) 1.17 & $1.17 \pm 0.01$ \\
\hline & (2) 1.33 & $1.33 \pm 0.05$ \\
\hline
\end{tabular}

\section{CONCLUSION}

This work discusses briefly the designing parameters and computational testing of the feasibility of a multi particle detection system that is based on three detection layers. Results show the prototype configuration is capable of detecting and localising thermal neutrons, fast neutrons and gamma-ray sources. The results from this study have been directly implemented to build the porotype imaging system which is at the moment under experimental investigation,

\section{REFERENCES}

1.

IAEA, IAEA Inicdents and Trafficking Database (ITDB) Incidents of nuclear and other radioactive material out of regulatory control, in 2018 Fact Sheet. 2018, International Atomic Energy Agency Vienna, Austria.

2. D. Reilly, N.E., H. Smith, and S. Kreiner, Passive Nondestructive Assay of Nuclear Materials, , N.E. D. Reilly, H. Smith, and S. Kreiner Editor. 1991: National Technical Information Service, U.S. Department of Commerce, Washington, DC,

3. Al Hamrashdi, H., S.D. Monk, and D. Cheneler, Passive Gamma-Ray and Neutron Imaging Systems for National Security and Nuclear Non-Proliferation in Controlled and Uncontrolled Detection Areas: Review of Past and Current Status. Sensors, 2019. 19(11): p. 2638.

4. Zelakiewicz, S., et al., SORIS-A standoff radiation imaging system. Nuclear Instruments and Methods in Physics Research Section A: Accelerators, Spectrometers, Detectors and Associated Equipment, 2011. 652(1): p. 5-9.

5. Ziock, K.P., et al. Source-search sensitivity of a largearea, coded-aperture, gamma-ray imager. in Nuclear Science Symposium/Medical Imaging Conference. 2004. Rome, ITALY.

6. Christian, J.F., et al. Nuclear material detection techniques. in SPIE Defense and Security Symposium. 2008. SPIE.

7. Budden, B.S., et al., A Cs2LiYCI6:Ce-based advanced radiation monitoring device. Nuclear Instruments and Methods in Physics Research Section A: Accelerators, Spectrometers, Detectors and Associated Equipment, 2015. 784: p. 97-104.

8. Gamage, K.A.A., M.J. Joyce, and G.C. Taylor, Investigation of three-dimensional localisation of radioactive sources using a fast organic liquid scintillator detector. Nuclear Instruments and Methods in Physics Research Section A: Accelerators, Spectrometers, Detectors and Associated Equipment, 2013. 707: p. 123126.

9. Soundara-Pandian, L., et al., Characterization of Large Volume CLYC Scintillators for Nuclear Security Applications. IEEE Transactions on Nuclear Science, 2017. 64(7): p. 1744-1748.

10. Polack, J.K., et al. Dual-particle imager for standoff detection of special nuclear material. in 2011 IEEE Nuclear Science Symposium Conference Record. 2011. Valencia, Spain.

11. PackEye Radiation Detection Backpack. [cited 2018 26/10/2018]; Available from:

https://www.thermofisher.com/order/catalog/product/ FHT1377.

12. Radiation Backpack Sentry. 2012 [cited 2018 26/10/2018]; Available from: 
https://www.bruker.com/fileadmin/user upload/8-PDFDocs/CBRNE Detection/Literature/Backpack-Brochureen.pdf.

13. Mascarenhas, N., et al., Results With the Neutron Scatter Camera. IEEE Transactions on Nuclear Science, 2009. 56(3): p. 1269-1273.

14. Al Hamrashdi, H., D. Cheneler, and S.D. Monk, Material optimization in dual particle detectors by comparing advanced scintillating materials using two Monte Carlo codes. Nuclear Instruments and Methods in Physics Research Section A: Accelerators, Spectrometers, Detectors and Associated Equipment, 2017. 869(Supplement C): p. 163-171.

15. Wraight, L.A., D.H.C. Harris, and P.A. Egelstaff, Improvements in thermal neutron scintillation detectors for time-of-flight studies. Nuclear Instruments and Methods, 1965. 33(2): p. 181-193.

16. SensL J-series. [cited 2018 01/11/2018]; Available from: https://sensl.com/products/j-series/

17. Knoll, G.F., Radiation detection and measurment. Fourth ed. 2010, New York, NY, USA: John Wiley and Sons.

18. Vanier, P.E. and L. Forman. Demonstration of a directional fast neutron detector. in IEEE Nuclear Science Symposium Conference Record, 2005. 2005.

19. Vanier, P.E., et al. Calibration and testing of a large-area fast-neutron directional detector. in 2007 IEEE Nuclear Science Symposium Conference Record. 2007. Honolulu, $\mathrm{HI}$, USA.

20. Macri, J.R., et al. The Fast Neutron Imaging Telescope (FNIT) - hardware development and prototype testing. in 2007 IEEE Nuclear Science Symposium Conference Record. 2007.

21. Todd, R.W., Nighting.Jm, and D.B. Everett, PROPOSED GAMMA CAMERA. Nature, 1974. 251(5471): p. 132-134.

22. Herzo, D., et al., A Large Double Scatter Telescope for Gamma Rays and Neutrons. Nuclear Instruments and Methods, 1975. 123(3): p. 583-597.

23. Kamae, T., N. Hanada, and R. Enomoto, PROTOTYPE DESIGN OF MULTIPLE COMPTON GAMMA-RAY CAMERA. leee Transactions on Nuclear Science, 1988. 35(1): p. 352-355.

24. Everett, D.B., et al. Gamma-radiation imaging-system based on compton-effect. in Proceedings of the Institution of Electrical Engineers-London. 1977. IET Digital Library: London, UK.

25. Singh, M. and R.R. Brechner, Experimental Test-Object Study Of Electronically Collimated SPECT. Journal of Nuclear Medicine, 1990. 31(2): p. 178-186.

26. Goorley, J.T.J., Michael R.; Booth, Thomas E.; Brown, Forrest, et al., Initial MCNP6 Release Overview - MCNP6 version 1.0. 2012.

27. Allison, J., et al., Recent developments in GEANT4. Nuclear Instruments \& Methods in Physics Research Section a-Accelerators Spectrometers Detectors and Associated Equipment, 2016. 835: p. 186-225.

28. Gozani, T., Active Nondestructive Assay of Nuclear Materials, Principles and Applications 1981, Washington DC: US Nuclear Regulatory Commission. 